\title{
INSEDIAMENTI, CHIESE E PORTI LUNGO IL BASSO ADIGE TRA VI E X SECOLO
}

\section{GIAN PIETRO BROGIOLO}

UDC: $726.54 \cdot 033 \cdot 1(450.34) " 05 / 09 "$ $27-526$

Original scientific paper

Manuscript received: 01. 02. 2016.

Revised manuscript accepted: 27. 03. 2016.

DOI: 10.1484/J.HAM.5.111360
G.P. Brogiolo Università degli studi di Padova Piazza Capitaniato 7 I-35139 Padova Italia

In the $6^{\text {th }}$ century the Adige river, which ran through the towns of Montagnana, Este, Monselice (Padua) and Brondolo (Chioggia, Venice), moved $20 \mathrm{~km}$ southward. Nevertheless, as extant churches and their liturgical furniture demonstrate, the most important towns survived this change, perhaps thanks to artificial waterways. During an update of the corpus of the early medieval churches in the Padua province, we studied these buildings systematically, using written and archaeological sources, as well as the detailed descriptions provided by the pastoral visits of the bishop Barozzi (1489). We hypothesize that a great number of recorded churches, as well as their liturgical furniture (see Vedovetto in this volume), can be dated prior the $10^{\text {th }}$ century, although this could only be confirmed by new archaeological data.

Key words: Adige river, Monselice, Este, early medieval church, CARE

A seguito degli sconvolgimenti ambientali, finora definiti solo in termini generali a partire dall'alluvione del 589 ricordata da Paolo Diacono, il corso del fiume Adige si è spostato verso sud, dove scorre tuttora con due rami che si formano a Badia Polesine: quello più settentrionale per Piacenza d'Adige, Anguillara, Borgoforte, Rottanova e Cavarzere sfocia nel mare Adriatico a sud di Brondolo (Chioggia); quello più meridionale, chiamato l'Adigetto, da Badia Polesine tocca Lendinara, Rovigo per poi risalire a Villadose, Lezze, Cavarzere e riunirsi al primo. Le trasformazioni ambientali fanno da sfondo ad un radicale cambiamento istituzionale che, alla fine del VI-inizi del VII secolo, vede la fine della città protostorica e romana di Este, sostituita da Monselice, nuovo capoluogo di una gastaldaga longobarda, mentre il territorio costiero dell'Adriatico (con i centri di Cavarzere, Loreo, Brondolo e Chioggia) rimane bizantino. Nonostante ciò, il vecchio tracciato dell'Adige, protostorico e romano, da Montagnana a Este, Monselice e Brondolo è rimasto attivo, grazie agli immissari che da nord si immettevano nel paleoalveo e a canali artificiali. Questo spiega la vitalità di centri come Montagnana, Megliadino, Este e Monselice presso i quali vi sono le chiese più importanti (fig.1), talora con elementi di arredo liturgico.

Nell'ambito di un aggiornamento del corpus delle chiese del padovano ${ }^{1}$, oltre ad utilizzare sistematicamente le consuete fonti scritte, archeologiche ed architettoniche, mi sono

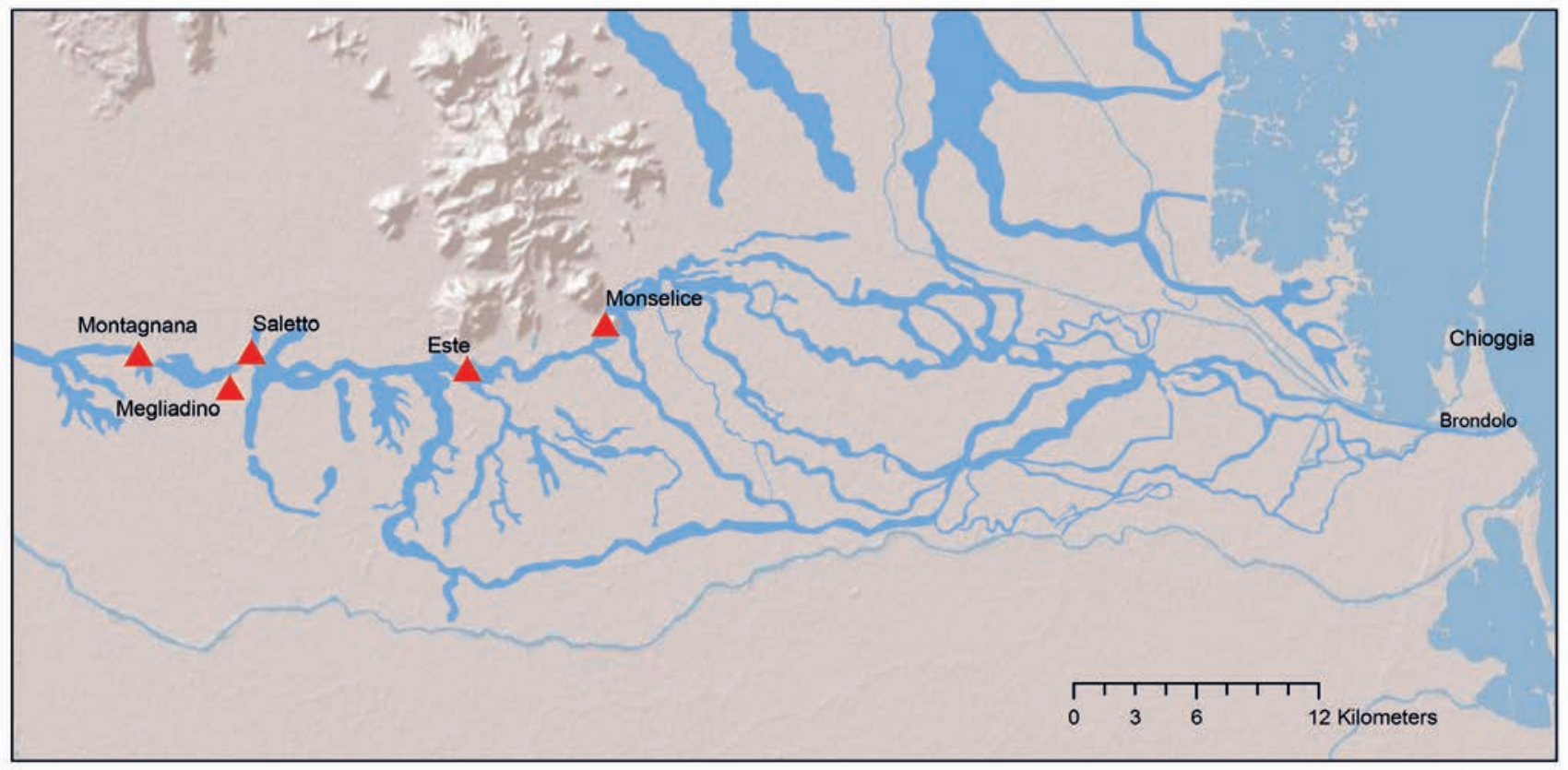

Fig. 1. Ubicazione delle chiese considerate in questo contributo, in rapporto ai percorsi dell'Adige.

${ }^{1}$ L'aggiornamento del Corpus (A. COLECCHIA, Padova, in G.P. BROGIOLO, M. IBSEN (a cura di), Corpus Architecturae Religiosae Europeae (saec. IV-X). Vol. II. Italia I. Province di Belluno, Treviso, Padova, Vicenza, Zagreb, 2009), è stato avviato nell'ambito delle attività di ricerca dell'Unità di Padova nei pro- 
servito delle dettagliate descrizioni contenute nella visita pastorale del vescovo Barozzi. Sebbene, per alcune chiese, sia necessaria una conferma archeologica, per altre vi è un'alta probabilità che nel 1489, anno della visita, fossero ancora nella loro originaria veste altomedievale e la proposta è di inserirle nella versione in rete del CARE.

\section{ESTE, CITT $\square$ RIDOTTA}

Este protostorica e romana era delimitata a sud dal fiume Adige, ad ovest da un antico percorso fluviale detto scolo di Lozzo, a nord ed est da un porto canale (denominato Sirone in età medievale) che si immetteva poi nell'Adige. A nord del canale, alle falde del colle, dominato ora dal castello carrarese, si estendeva una delle necropoli della città paleoveneta e romana, mentre sulla sommità sorgeva forse un luogo di culto. Appena delineate sono le vicende della città romana, mentre di Este tardoantica non si conosce quasi nulla, il che ha avvalorato la conclusione, ribadita anche recentemente, che "il centro urbano si sia avviato verso un graduale declino già a partire dal II sec. d.C., tramontando definitivamente nel corso del IV sec."2 Conclusione che va peraltro mitigata, perlomeno in attesa di ricerche sistematiche, in quanto numerosi sono gli indizi di una continuità dell'assetto idrografico e di occupazione di almeno cinque settori urbani (fig.2).

Sulla sommità del colle che domina Este, nel 1914 è stato parzialmente scavato un possente edificio rettangolare di m 20,30 x 18 (datato dallo scavatore, Alfonso Alfonsi, tra VI e VII secolo 3 ). In appoggio al lato nord dell'edificio è stata costruita una torre trapezoidale, con muri dello spessore da $\mathrm{m}$ 2,35 a m 3, la cui forma è tipica dell'architettura militare bizantina. Potrebbe dunque essere stata costruita nel contesto della guerra greco-gotica o in quello, immediatamente posteriore, della difesa dell'Adige tra l'invasione longobarda (569) e la conquista di Monselice nel 602. L'ipotesi più probabile, come per altre torri altomedievali erette lungo il percorso dell'Adige ${ }^{4}$, è che l'edificio rinforzato dalla torre pentagonale avesse una funzione difensiva accanto ad altri compiti di natura pubblica, in relazione al porto sul fiume Sirone, suggerito dal ritrovamento di una banchina spondale ai piedi del castello e dalle fonti scritte nel basso medioevo.

Oltre al ridotto sul colle, a nord del porto-canale, cosa rimaneva in età altomedievale della città romana? Gli scavi non hanno rinvenuto (o riconosciuto) fasi altomedievali, salvo il ritrovamento, nella domus del Serraglio Albrizzi,

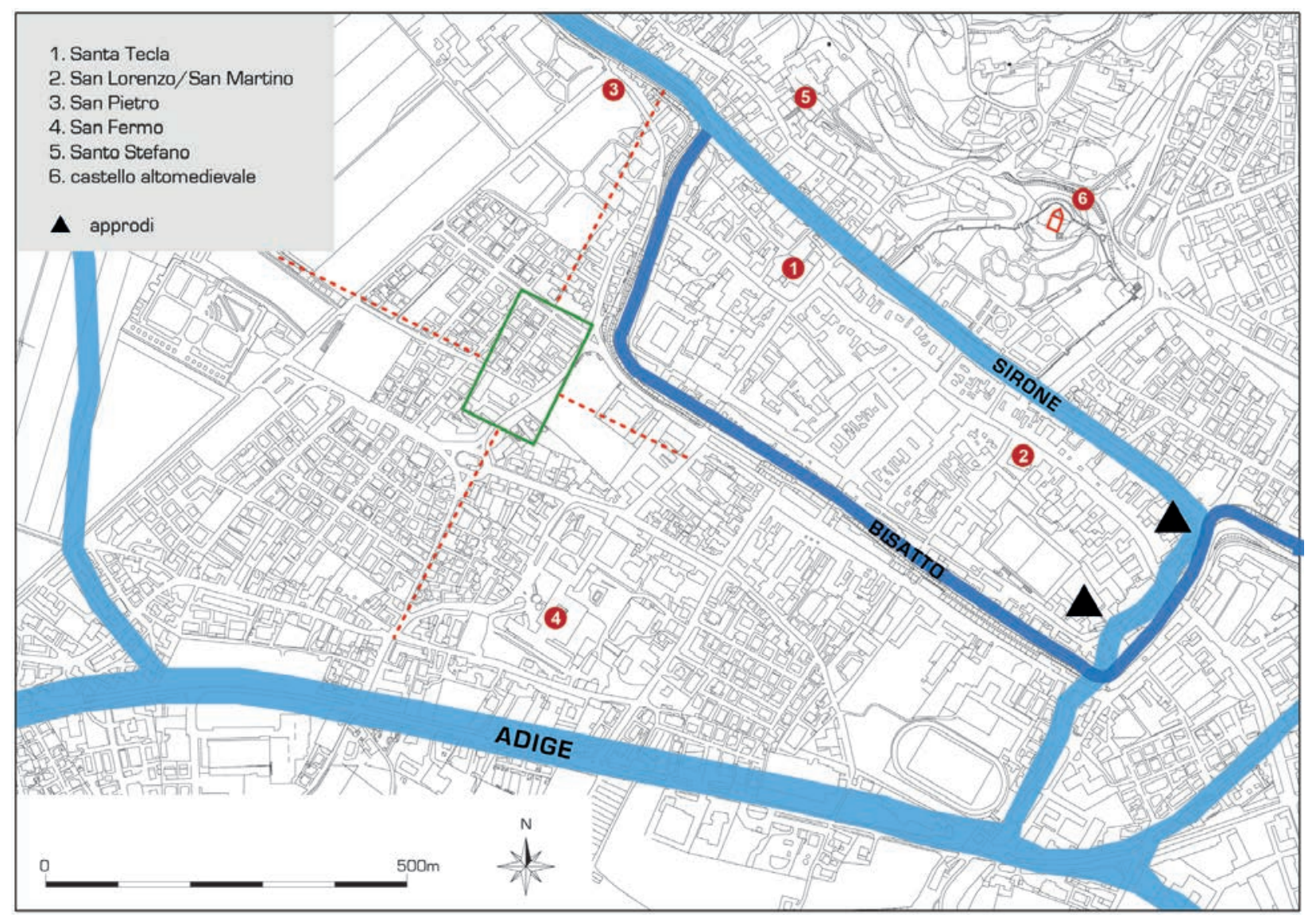

Fig. 2. Este con l'idrografia antica (vecchio Adige e Sirone) e il successivo canale Bisatto (1139) che delimita l'abitato medievale all'interno del quale si trovavano le chiese di Santa Tecla e San Lorenzo/San Martino, mentre erano all'esterno Santo Stefano, San Pietro e San Fermo e, sulla sommità del colle, un grande edificio protetto da una torre pentagonale.

getti PRIN 2010 - 2011 (2010H8WPKL_010) "Storia e archeologia globale dei paesaggi rurali in Italia tra Tardoantico e Medioevo. Sistemi integrati di fonti, metodi e tecnologie per uno sviluppo sostenibile" e PRAT - Ateneo di Padova 2012 (CPDA128591/12) "Paesaggi, Architetture e identità locali nel Medioevo: nuove procedure di catalogazione e analisi archeologica".

${ }^{2}$ C. Maratini in C. MARATINI, M. VIGATO (a cura di), Uomini, terre e acque, Este, 2014, p. 52.

3 Degli scavi esistono, presso l'archivio del Museo di Este, i giornali e una relazione dattiloscritta dell'Alfonsi datata 29 gennaio 1919, dalla quale hanno tratto alcune notizie A. MONETI, A. DRAGHI, Un secolo di archeologia medievale nel castello di Este, in Archeologia Medievale, XXIV (1997), pp. 173-182. ${ }^{4}$ G.P. BROGIOLO, Le torri altomedievali lungo l'Adige, in Suadente nummo vetere. Studi in onore di Giovanni Gorini, Padova, 2016, pp. 459-474. 
di una sepoltura femminile di epoca ostrogota con orecchini a poliedro in oro decorati con almandini ${ }^{5}$. E tuttavia la topografia cristiana, tutta impostata nel settore orientale della città romana e alle falde del colle, suggerisce una vitalità del centro antico, seppur ridotto in superficie e fortificato, ma ancor legato ad un sistema idrografico che lo collegava al mare.

Delle cinque chiese che possiamo ipotizzare altomedievali (Santa Tecla, San Martino, Santo Stefano, San Pietro, Santi Fermo e Rustico), la più interessante è indubbiamente la prima, ricordata per la prima volta, come pieve, nel 1034 e con il titolo di Santa Tecla in atti successivi ${ }^{6}$. Integralmente ricostruita dopo il crollo delle volte nel 1689, ne abbiamo alcune testimonianze materiali, mentre nella visita del Barozzi (1489) ne viene puntualmente descritta la pianta articolata in cinque navate (quattro parallele e una ortogonale), tutte provviste di altari, con le domus dell'arciprete e dei canonici a nord, il battistero e il campanile a sud. Delle cinque navate, le tre verso nord (Santa Tecla II) credo siano riferibili ad una fase romanica della fine dell'XI secolo che discuterò in un altro contributo. Le altre due navate, ortogonali tra loro e più piccole e collegate, nel 1489, ad un battistero (in origine un sacello esterno), sono verosimilmente attribuibili ad un complesso precedente (Santa Tecla I), fondato, secondo la leggenda riportata dal Barozzi, da San Prosdocimo, circostanza alla quale si devono

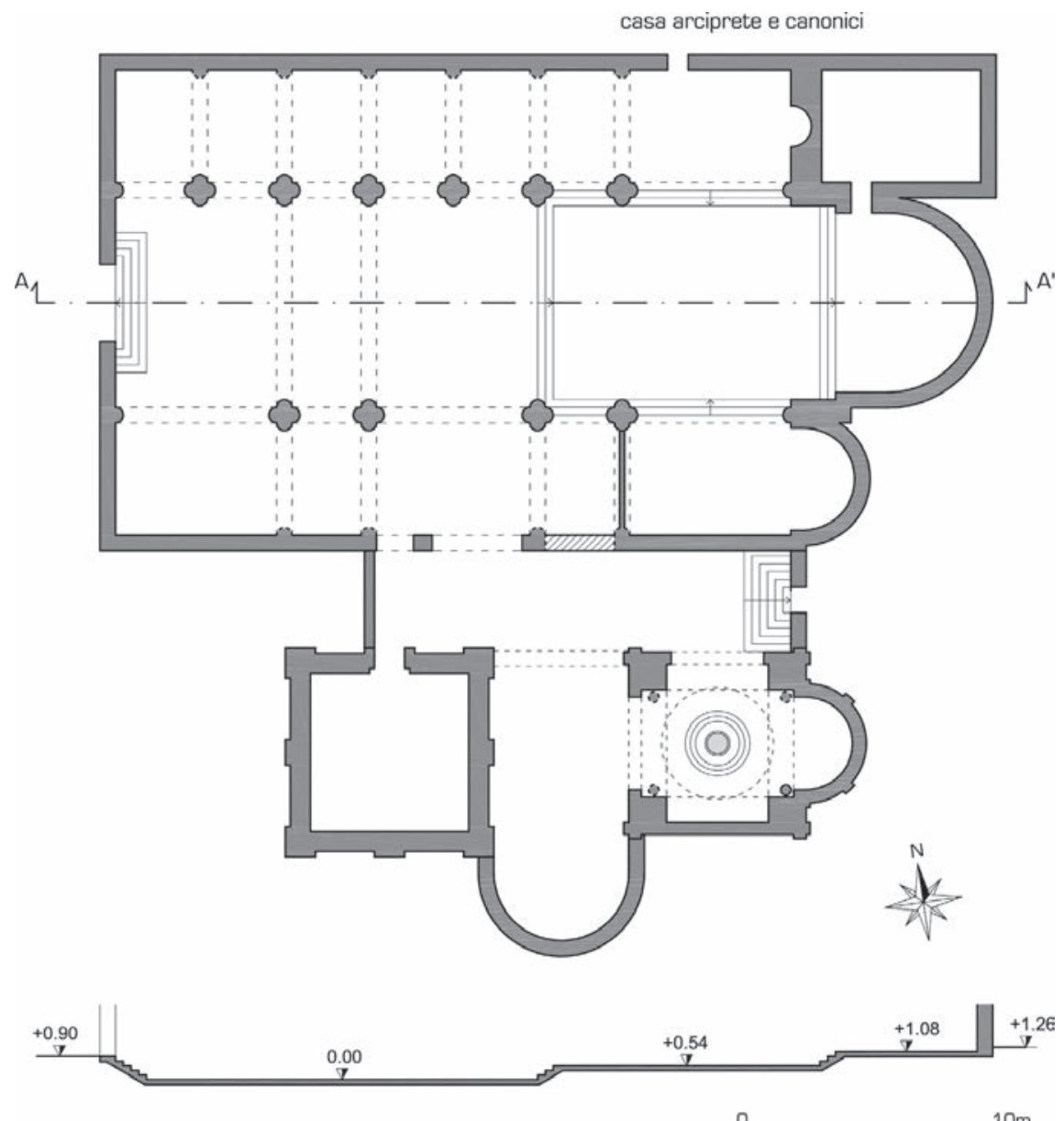
probabilmente la conservazione e il collegamento monumentale alla chiesa romanica. Quantunque il riferimento al protovescovo padovano del IV secolo sia un topos, sul quale non si può fare affidamento, l'ipotesi di un'origine antica può essere proposta.

Ma procediamo con ordine, soffermandoci anzitutto sulle quote pavimentali dei due complessi. Per entrare, da ovest, nella navata centrale di Santa Tecla II, nel 1489 bisognava scendere 5 gradini per un totale di ca. $90 \mathrm{~cm}$ (2 piedi e mezzo). Per accedere alla quarta navata i gradini erano invece sette, ovvero m 1,25 calcolando la medesima alzata di $18 \mathrm{~cm}$. Alcuni scavi attorno alla chiesa hanno individuato i livelli romani a ca. 1.50 di profondità. Inoltre, nel 1583, all'esterno della chiesa vennero trovate, alla profondità da 4 a 6 piedi (da un metro e mezzo a due) grandi lastre di pietra di Verona (una delle quali misurava $\mathrm{m} \mathrm{1,63} \mathrm{x} \mathrm{0,68} \mathrm{X} \mathrm{o,25),} \mathrm{dunque} \mathrm{un}$ piano pavimentale lastricato che può essere messo in relazione con quanto scoperto, nel 1724, a fianco del campanile, dunque nell'area di Santa Tecla I: un edificio con pavimento musivo e i resti di una statua equestre in bronzo dorato ${ }^{7}$. Nel 1997, un altro pavimento musivo di un edificio, significativamente con impianto ortogonale rispetto a Santa Tecla, è

venuto in luce nella vicina via Zanchi, ad una profondità di due metri ${ }^{8}$, ma è da tener conto un provabile innalzamento delle quote di calpestio dalla fine del '5oo ad oggi.

L'ipotesi è dunque che la prima chiesa sia stata costruita nello spazio occupato in precedenza da un edificio pubblico romano, ma non il foro, ubicato a sud ovest ${ }^{9}$.

Non sappiamo invece a quale fase siano riferibili le fondamenta della chiesa in conci di pietra veronese ed istriana molti dei quali lavorati ${ }^{10}$ e dunque con ogni probabilità recuperati da questo edificio.

Il battistero di San Giovanni Battista, descritto dal Barozzi è stato parzialmente documentato, nel 1986, da uno scavo che ha messo in luce "metà del muro absidale circolare, il pilastro destro e una parte della parete destra, tutti costruiti con mattoni romani e poche aggiunte medievali" ${ }^{11}$. L'edificio aveva pianta quadrangolare di ca. $\mathrm{m} 7 \mathrm{per}$ lato, con abside semicircolare ad oriente del diametro di $\mathrm{m}$ 3,57, provvista di un paio di lesene esterne. Quattro pilastri sostenevano le arcate su cui si impostava la volta circolare alleggerita da quattro semicalotte agli angoli. Tra l'abside e il pilastro di destra, nel 1986, è stata individuata anche la

\footnotetext{
${ }_{5}$ C. MARATINI, M. VIGATO, Uomini..., p. 53, nota 91.

${ }^{6}$ A. GLORIA (a cura di), Codice Diplomatico Padovano: dal secolo sesto a tutto l'undecimo, Venezia, 1877, n. 130.

${ }^{7}$ I. ALESSI, Ricerche istorico-critiche delle antichità di Este, Este, 1776, pp. 247-248.

${ }^{8}$ B. COGO, Alla scoperta del duomo di Este, Este, 2012, p. 148.

${ }^{9}$ C. TAGLIAFERRO, L'organizzazione viaria urbana di Este, in Archeologia Veneta, XXXVIII (2015), pp. 53-61.

${ }^{10}$ Cronaca del Davò, citata da I. ALESSI, Ricerche..., p. 484.

${ }^{\text {" } B . ~ C O G O, ~ A l l a ~ s c o p e r t a . . ., ~ p p . ~ 139-140 ~ e ~ f o t o ~ d i ~ p . ~} 141$.
} 
base di una colonna ${ }^{12}$, per cui possiamo ipotizzare che per simmetria ve ne fosse almeno un'altra contrapposta, se non quattro agli angoli. Il battistero era collegato, verso ovest, tramite un'arcata di ben $\mathrm{m}$ 3,93, alla quinta navata descritta dal Barozzi (in realtà un atrio rettangolare, con abside a sud, costruito tra il battistero e il campanile), verso est, da un'arcata identica, alla quarta navata. All'interno del battistero il fonte battesimale si trovava al centro su un podio circolare di due gradini e al di sopra di un cippo sul quale erano scolpiti Adamo ed Eva e un santo con un rotolo in mano, forse il Battista. Le sculture sono probabilmente di XI secolo $^{13}$, ma i confronti per il battistero ci portano in un'altra direzione, tutti della prima metà del VI secolo, avvero ai sacelli di Opilione a Santa Giustina di Padova, di San Felice e Fortunato di Vicenza, di San Gregorio di Rimini e, più in generale, con i sistemi voltati delle chiese bizantine di quel periodo. E identica è la disposizione del sacello, rispetto alla chiesa, almeno per Este, Padova e Vicenza.

In conclusione credo si possa con cautela avanzare l'ipotesi che Santa Tecla I fosse una chiesa a tre navate e unica abside con complessive misure interne di $\mathrm{m}$ 13,70 x 16,10, alla quale si addossava un sacello con antistante atrio. Fondata su un edificio pubblico, solo $25 \mathrm{~cm}$ al di sopra dei piani di calpestio della città romana, la committenza, come nel caso di Santa Giustina di Padova, eretta all'interno di un cimitero della città, va dunque ricercata nell'ambito pubblico, forse in relazione con la costruzione, sulla sommità del colle (di fronte a Santa Tecla), del nuovo centro direzionale e difensivo. In origine con funzione funeraria (in un'area urbana divenuta periferica) Santa Tecla sarebbe stata convertita in "cattedrale" provvisoria agli inizi del XII secolo, quando ad Este si trasferisce il vescovo riformatore Sinibaldo, cacciato da Padova dal filoimperiale Pietro. Proprio a Sinibaldo sarebbero da attribuire la ricostruzione romanica della chiesa, progettata in modo da enfatizzare il suo collegamento con gli edifici precedenti ${ }^{14} \mathrm{e}$ in particolare con il sacello trasformato in battistero.

Questa conclusione, da sottoporre ad ulteriori verifiche, trova un altro supporto in una notizia, riferita dal vescovo Barozzi nella visita pastorale all'allora monastero di San Martino: secondo "antichi privilegi", in origine era una chiesa plebana dedicata a San Lorenzo e aveva sotto di sé la cappella della Santa Trinità di Pra'; dopo un ampliamento e a quel titolo aggiunse quello di s. Martino, che divenne infine l'unico titolare. Un codice della fine del XVI secolo ci informa poi che la chiesa di San Martino a Longobardis (...) constructa fuit ${ }^{15}$. Divenuta monastero, nella prima metà dell'XI secolo venne donata dal vescovo Burcardo al cenobio padovano di Santo Stefano ${ }^{16}$. Il Barozzi la descrive a pianta quadrata di $\mathrm{m} \mathrm{13,90}$ (8 passi) di lato, per un'altezza di $\mathrm{m}$ 6,95 (4 passi), con ad oriente un'abside, pure quadrata, di $\mathrm{m} 6,95$ (4 passi) di lato, alta $\mathrm{m}$ 4,64 (13 piedi) fino all'inizio dell'arco, che nella parte mediana ha forma di mezza sfera.

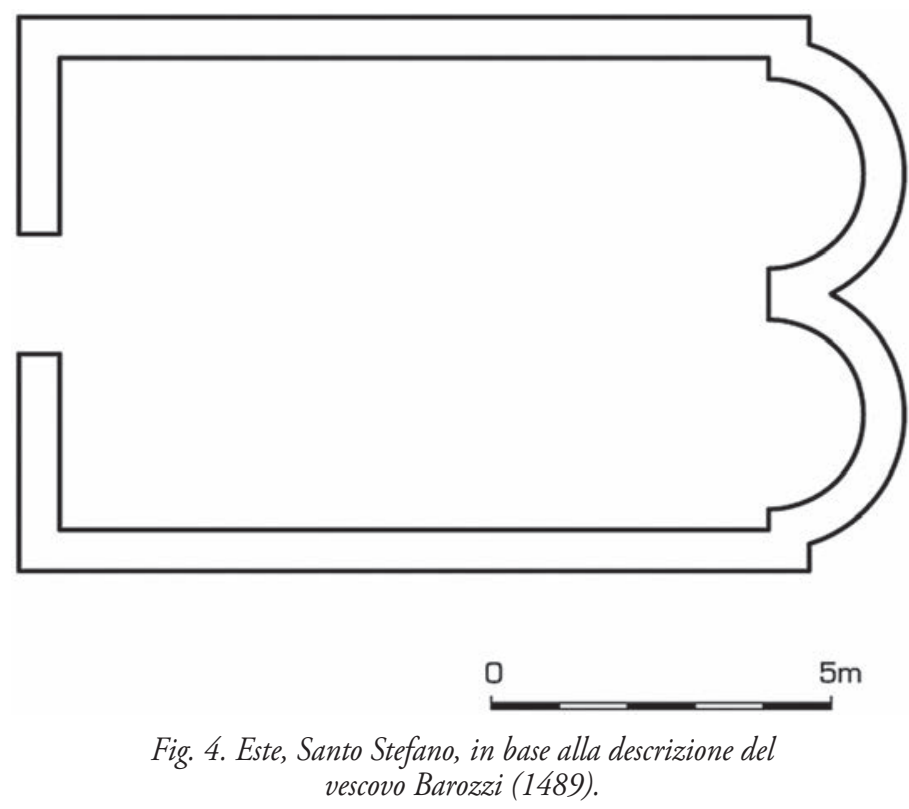

Quest'abside non era esattamente al centro ma spostata verso nord. Vi era infatti una seconda abside, più piccola, appartenente, secondo il Barozzi, alla prima chiesa. Tra le due absidi, la parete era sufficientemente ampia da ospitare un terzo altare. Divideva la parte dei maschi da quella delle donne un "podio" alto $\mathrm{m}$ 5,71 (16 piedi), verso oriente in laterizi, verso occidente con tre colonne, su cui si impostava una volta, alte $\mathrm{m} \mathrm{2,85}$ (8 piedi), comprese le basi e i capitelli. La descrizione si riferisce alla sola parte orientale della chiesa, caratterizzata da una tecnica muraria ad opera listata della prima metà del XIV secolo e non all'atrio che si sviluppava verso ovest. Il lato meridionale dell'atrio, che si conserva tuttora, ha paramento in laterizi romani di riuso ed è scandito da lesene, peculiarità che lo fanno riferire alla prima chiesa di San Lorenzo, il cui il cui termine ante quem è costituito dagli elementi di arredo liturgico dell'VIII secolo (Vedovetto in questo volume). Uno scavo recente, del quale non ho potuto vedere la documentazione, dovrebbe aver documentato la sequenza.

Una terza chiesa, dedicata a Santo Stefano, sorgeva ai piedi del colle, a nord del fiume Sirone, in area cimiteriale romana, il che ne suggerisce un'origine come basilica funeraria suburbana. Nella Visita del Barozzi ne vengono riportate la dimensione di $\mathrm{m} 6,95 \times 10,42$ ( 4 × 6 passi) con altezza di m 6,95 (4 passi) e la presenza di due absidi semicircolari ad oriente, particolare questo che potrebbe riferirla ad una fase altomedievale.

Attorno ad altre due chiese suburbane si erano sviluppati dei borghi: San Pietro presso il fiume Sirone, mentre quella dedicata a San Fermo era sul lato opposto presso il ponte sull'Adige, nel borgo di Settabile. Nel 1123 dipendevano entrambe dal monastero della Vangadizza ${ }^{17}$.

\footnotetext{
${ }_{12}$ B. COGO, Alla scoperta..., p. 141.

$13 \mathrm{P}$. Vedovetto in questo volume.

${ }^{14}$ Il collegamento è stato ottenuto tramite due arcate di maggior ampiezza nel divisorio tra navata centrale e laterale sud e con un collegamento tra questa e la quarta navata (che ho ipotizzato ricalchi quella della prima chiesa) attraverso un'ampia arcata e una finestra, dalla quale si poteva vedere il celebrante nella terza navata.

15 “Codice Extravagante”, presso Arch. della Magnifica Comunità di Este, A 58, c. 18, citato da Cogo, Alla scoperta..., p. 40.

${ }^{16}$ La donazione comprende omnia jura ac decimas del monastero di San Martino cum tota sua pertinentia de plebe Adeste: I. Alessi, Ricerche ..., p. 485 .

${ }^{17}$ I. ALESSI, Ricerche..., p. 508.
} 

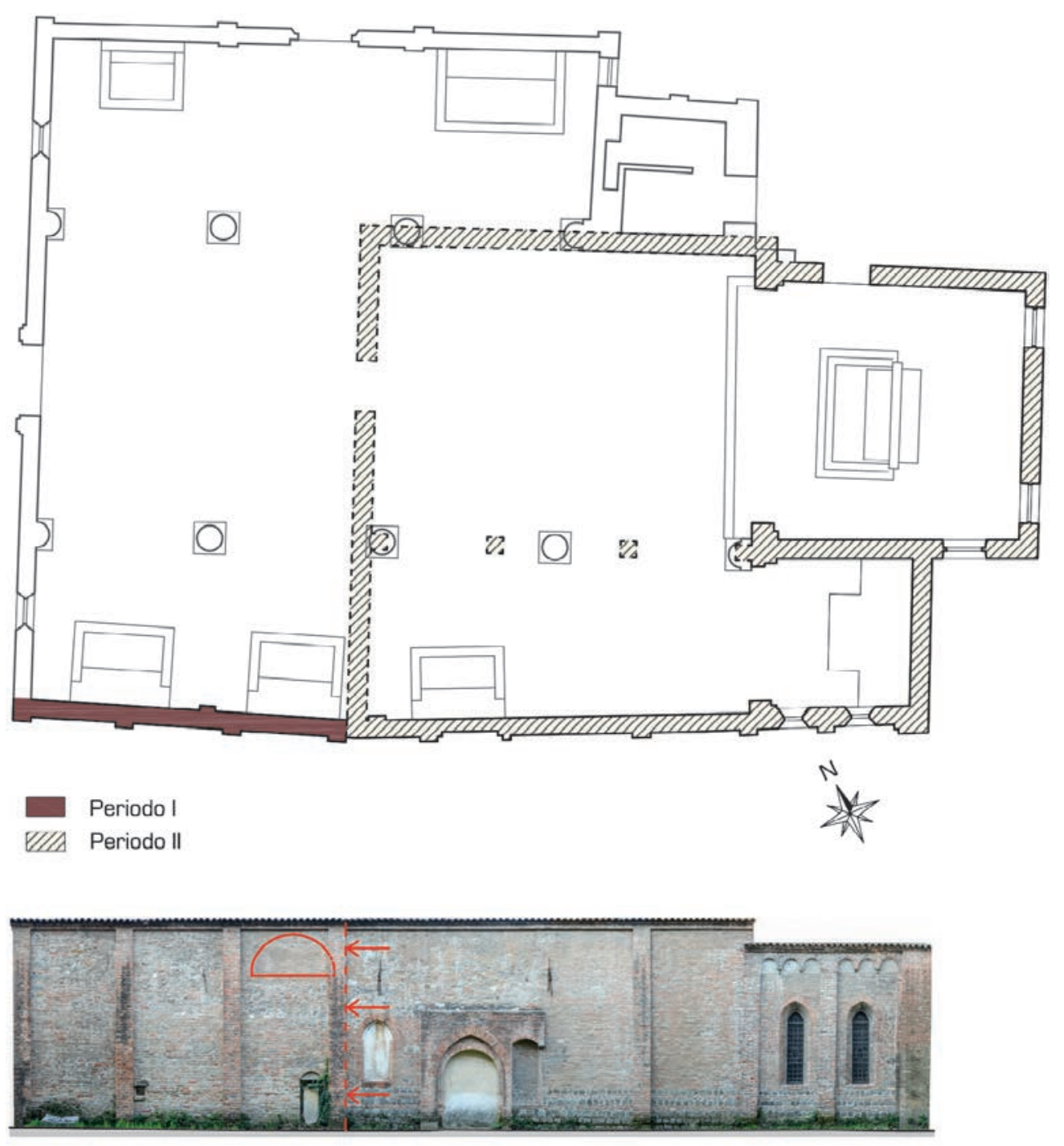

parete sud

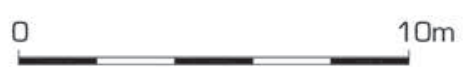

Fig. 5. Este, San Lorenzo/San Martino, pianta ricavata dalla descrizione del vescovo Barozzi (1489) e fotopiano della parete sud.

In conclusione, secondo un modello di città altomedievale ridotta e policentrica ${ }^{18}$, ad Este sul colle si era insediato un centro direzionale e di difesa, dal quale si svilupperà poi un ampio castello fino al fiume Sirone. In piano, due isole di insediamento erano sorte attorno alle chiese di San Lorenzo, non lontano dagli approdi fluviali e antica battesimale secondo il Barozzi, e Santa Tecla, fulcro della "villa" medievale. Altre due, legate alle chiese di San Pietro e San Fermo, si erano formate presso gli attraversamenti dei fiumi Sirone e Vecchio Adige.

\section{MONSELICE: LA TOPOGRAFIA CRISTIANA DI UN NUOVO CAPOLUOGO (VI-X SECOLO)}

Il castello di Monselice, capoluogo del vecchio distretto di Este dopo la conquista longobarda del 6o2, sorgeva sulla sommità di un colle isolato degli Euganei che dominava l'Adige e la pianura circostante nelle direzioni di Padova, Adria e Ravenna. Era dunque in una posizione strategica sia per difendere Ravenna, capitale gota, sia per proteggere i terri- tori rimasti bizantini dagli attacchi provenienti da Vicenza e Verona, città in mano longobarda fin dal 569. Secondo Aldo Settia la fondazione di Monselice si colloca nell'ambito del consolidamento dei possessi bizantini sulla costa adriatica, al tempo degli esarchi Smaragdo (585-589), Romano (589-598) o Callinico $(598-603)^{19}$. Non così precisa può essere l'archeologia, che si deve accontentare di situarne, e per di più con cautela, la fondazione tra la guerra greco-gotica e la seconda metà del VI secolo. Quale ne sia l'origine, è indubitabile fosse strategica nel controllo bizantino, tra 569 e 602, sia del percorso fluviale tra Verona, Este, Monselice, Pernumia, Civé e la laguna, sia delle strade nord sud che lo attraversavano. Paolo Diacono racconta che nel 572, dopo l'uccisione del re Alboino nel palazzo di Verona, la moglie Rosmunda el'amante Elmichi, che ne avevano tramato la morte, vengono messi in salvo da una nave bizantina che li porta a Ravenna.

Gli scavi, condotti tra il 1989 e il 1996 sul colle di Monselice ${ }^{20}$, hanno permesso di: (a) riconoscere la possente cinta muraria in grandi blocchi di trachite che correva a mezzaco-

${ }^{18}$ G.P. BROGIOLO, Le origini della città medievale, Mantova, 2015 (2.a edizione).

${ }^{19}$ A.A. SETTIA, Monselice nell'alto medioevo, in A. RIGON (a cura di), Monselice: storia, cultura e arte di un centro minore del Veneto, Treviso, 1994, pp. 83-99.

${ }^{20}$ G.P. BROGIOLO, Ricerche archeologiche su Monselice bizantina e longobarda, in A. RIGON (a cura di), Monselice: storia...pp. 47-63. 


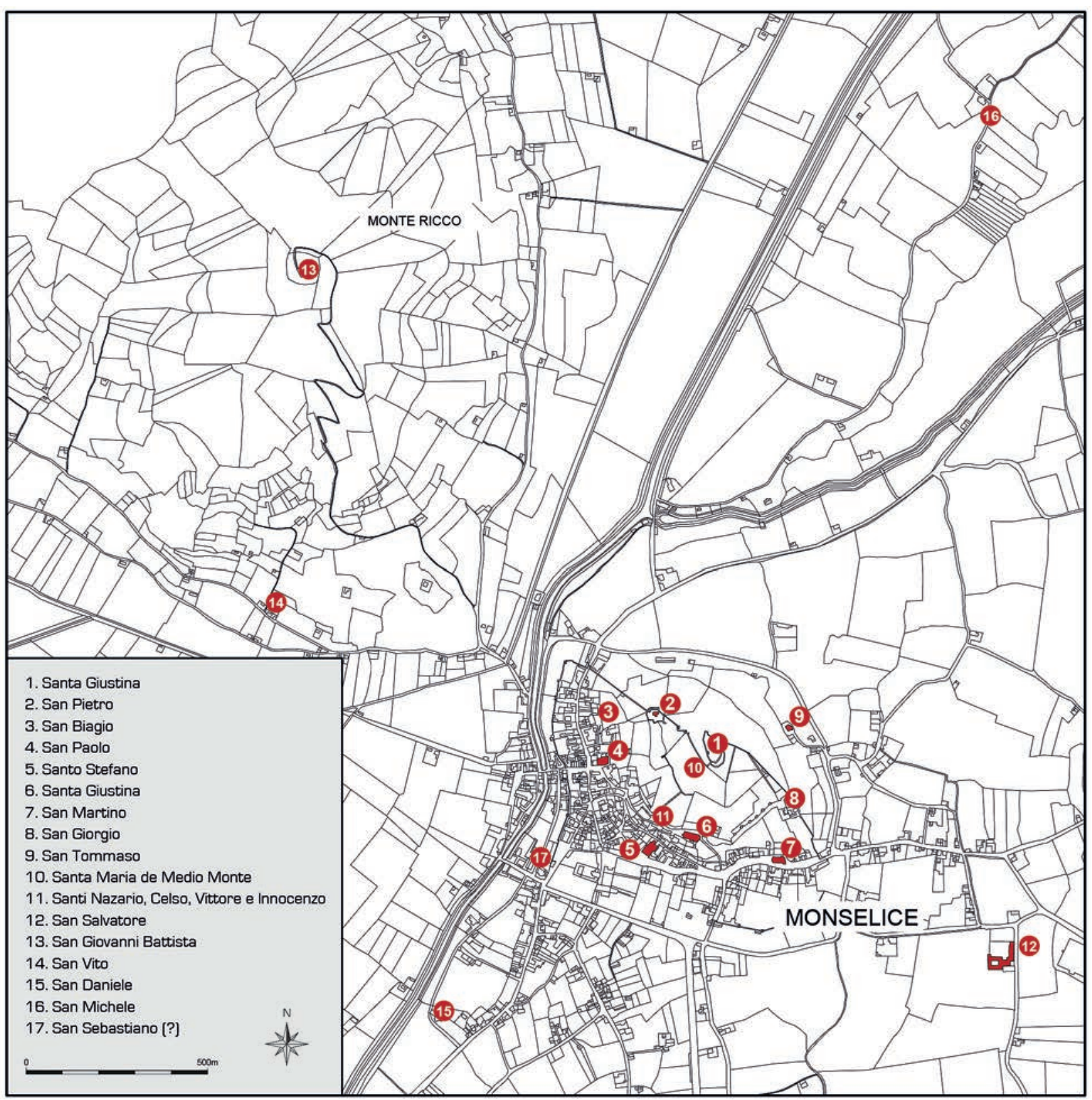

Fig. 6. Monselice, le probabili chiese altomedievali.

sta del versante meridionale, salendo poi sulla sommità; (b) identificare e scavare una torre della cinta, presso la quale, nella prima metà del VII secolo, sono state deposte sepolture con corredi longobardi, un paio delle quali di armati; (c) individuare sulla sommità i resti della chiesa di Santa Giustina, testimoniata dal 968, ma con ogni probabilità coeva al castello; (d) recuperare ceramiche a stampiglia e stralucido e numerosi frammenti di anfore di VI-VII secolo che suggeriscono una cultura materiale identitaria longobarda, ma aperta a scambi con la vicina area adriatica; (e) mettere in luce, sul versante meridionale del colle, stratificazioni altomedievali che confermano come l'abitato si fosse esteso fino al pedemonte, dove nell'VIII secolo. venne costruita la chiesa di San Paolo.

In mancanza di informazioni dettagliate sull'evoluzione urbanistica di Monselice tra il VII e il X secolo, la sua importanza si può per ora dedurre dalla topografia cristiana che comprendeva una quindicina di chiese: tre, forse quattro, compresa la pieve di Santa Giustina, si trovavano all'interno della prima cinta che dalla sommità scendeva a mezzacosta; due probabilmente tra la prima e una seconda cinta difensiva; tre nel pedemonte, mentre le altre erano sorte nella campagna circostante e sul vicino monte Ricco (fig. 6).

\section{(a) Le chiese all'interno della prima cinta}

La pieve di Santa Giustina, ricordata per la prima volta nel luglio del 968 assieme al suo arciprete Martino, è stata demolita, negli anni 'zo del Duecento, per ordine dell'imperatore Federico II che vi fece costruire il mastio ${ }^{21}$. Davanti a questo, è stato messo in luce l'angolo sud ovest della chiesa, impostato direttamente sulla roccia livellata. Le murature hanno lo spessore di $\mathrm{m} 1,70$, risultato peraltro di più fasi costruttive, la più recente delle quali presenta verso l'esterno un

\footnotetext{
${ }^{21}$ A. GLORIA (a cura di), Codice..., n. 51. Nella Visita Barozzi del 1489, si ricorda il decreto del vescovo di Padova Giovanni emanato l'11 ottobre del 1256 e la successiva conferma papale; cfr. A. DONDI DALL'OROLOGIO, Dissertazioni sopra l'istoria ecclesiastica padovana, VII, Padova, 1813, p. 74; A. GLORIA, Il territorio padovano illustrato, Padova, 862, v. IV, p. 133.
} 
paramento in grossi conci di trachite squadrati. Non è chiaro quale funzione avesse questa struttura: la fondazione, assai consistente, farebbe pensare ad una torre campanaria, ma l'andamento semicircolare all'interno potrebbe forse riferirsi ad un edificio a pianta centrale, adatto ad un fonte battesimale. Sul lato opposto, oltre il mastio, sono stati riconosciuti due tratti di muratura con andamento curvilineo, attribuiti ad un'abside semicircolare. Tessere di mosaico negli strati di demolizione suggeriscono una pavimentazione musiva della chiesa, alla quale sono riferibili anche otto sepolture a cassa senza corredo.

Oltre alla Pieve, all'interno del castello del VI secolo, sono ricordate due chiese dedicate, rispettivamente, a Santa Maria e a San Pietro. Nell'angolo sud est della cinta, sempre a metà del colle, gli scavi hanno poi documentato una torre d'angolo, forse trasformata, nella prima metà del VII secolo, in cappella funeraria, decorata con affreschi, di un'area cimiteriale dove vennero sepolti i longobardi provvisti di corredo di armi ${ }^{22}$.

Nella visita pastorale del vescovo Barozzi (1489), si precisa che una chiesa dedicata a Santa Maria si trovava a mezzacosta, verso occidente, ed era stata ricavata da una torre (in realtà una "casa forte", viste le dimensioni) di m 8,69 di lato (5 passi). Si conservavano allora le vestigia di una sola navata, rispetto alle tre originarie suddivise da tre archi, impostati su due colonne (arcus tres innixos columnis duabus lapideis, hinc atque inde, parieti). In una foto di fine ' 800 , si vedono: (a) la muratura con paramento in laterizi, salvo nella parte alta dove è almeno in parte in pietre; (b) un portale con arco bardellonato poggiante su un capitello; (c) una colonna con capitello tronconico all'incirca al centro dell'aula. Sempre secondo il visitatore, le pareti erano decorate da affreschi che testimoniavano l'importanza della chiesa. Il Cognolato, alla fine del '7oo, ricorda che alla chiesa, dedicata a Maria Vergine, era annesso un monastero femminile; ormai abbandonato, se ne vedevano ancora le sepolture da lui attribuite alle suore ${ }^{23}$.

Subito dopo Santa Maria, il Barozzi, scendendo dal colle verso il piano ma sempre all'interno della prima cinta, visita la chiesa di San Pietro, costruita presso una seconda torre che dalla chiesa aveva assunto il nome. Il Cognolato sostiene che, ai suoi tempi, di questa chiesa vi erano "antiche memorie del secolo IX"24; quel che è certo è che nel 1013 dipendeva dal Monastero della Vangadizza ${ }^{25}$. Ben proporzionata (con la lunghezza pari al doppio della larghezza e a quattro volte il diametro dell'abside), misurava m 6,95 x 13,90 (passi 4 x 8 ) ed era provvista di un'abside semicircolare del diametro di $\mathrm{m} 3,48$ ( 2 passi) con pari altezza fino all'inizio dell'arco. Il visitatore precisa che l'abside era divisa dalla navata da una recinzione presbiteriale alta un metro e sette centimetri e provvista, evidentemente in corrispondenza dell'accesso, di un arco centrale sorretto da due colonne (pariete lapideo pedum tres et columnis duabus arcu lapideo superposito). Secondo lui si trattava di un apprestamento liturgico realizzato more greco, evidentemente ignorando la sua diffusione in età tardo antica e altomedievale. Della recinzione in marmo, che offre un termine ante quem (o forse meglio ad quem) per la chiesa, un pezzo, databile all'VIII secolo ${ }^{26}$, potrebbe essere finito nei depositi comunali.

Se la pieve risale probabilmente alla fase di fondazione del castello, negli altri luoghi di culto siamo di fronte ad una dismissione di edifici e spazi pubblici che vengono destinati ad insediamenti religiosi. Con ogni probabilità siamo ancora in una fase altomedievale iniziale (VII-VIII secolo?), ma in assenza di dati di scavo non sappiamo in che relazione fossero con l'abitato sviluppatosi all'interno del castello e tra la prima e la seconda cinta.

\section{(b) chiese sui versanti}

Una strada collegava San Pietro, alla "mansione", un edificio pubblico eretto ai piedi del colle nel quale i fratelli, entrambi marchesi, Alberto Azzo I e Ugo, antenati della casa estense, presiedettero nel 1013, unitamente al conte padovano Todello, un placito riguardante la proprietà della corte di Petriolo ${ }^{27}$. L'ubicazione esatta della "mansione" è incerta; è però probabile si trovasse all'interno del giro di mura che si intravvede nella cartografia storica a monte di Ca' Marcello (nome della famiglia veneziana che acquistò il palazzo imperiale costruito alla fine del XII secolo). Il vescovo Barozzi, dopo aver visitato San Giorgio, risale infatti verso nord lungo la strada all'esterno delle mura (juxta muros) che porta alla Rocca. E' dunque probabile che la mansio sia da ricercare in uno dei tre edifici medievali che si trovavano a ridosso delle mura (due, fortemente restaurati, sono conservati in alzato, di un terzo si vede la parte inferiore del perimetrale di fronte di Ca’ Marcello).

La cappella dedicata ai Santi Nazaro, Celso, Vittore e Innocenzo, secondo il Barozzi, era alla distanza di un tiro di freccia dalla pieve (quella ricostruita sul versante meridionale nella seconda metà del XIII secolo), a monte della strada che portava dalla pieve a San Paolo. E' possibile si trovasse dove si vedono ora le facciate di alcuni edifici medievali, che fanno da sostruzione al giardinetto soprastante, adiacente alla strada che da Ca' Marcello porta alla pieve. Costruita contro il declivio, seminterrata sul lato settentrionale mentre gli altri lati erano fuori terra, misurava $m$ 6,95 x 5,20 (4 x 3 passi) ed era alta $m$ 5,20 ( 3 passi). Nell'abside ad oriente vi era un altare a cippo costituito da una base quadrangolare con sopra una piccola lastra (stipes columna quadrangula et lapis superior parvus); aveva inoltre due finestre obscuras et informes (una nell'abside, l'altra a sud) e due porte (una ad ovest, l'altra a sud).

\footnotetext{
${ }^{22}$ G.P. BROGIOLO, Ricerche...

${ }^{23} \mathrm{G}$. COGNOLATO, Saggio di memorie della terra di Monselice di sue sette chiese del santuario in esse aperto ultimamente, Padova, 1794, p. 52.

${ }^{24}$ Già in rovina nel 1476 (T. LUSIANI, Notizie sul monastero di S. Pietro di Monselice nei secoli XV-XVII, in Atti e memorie del Sodalizio Vangadiciense, 1 (1972-1973), p. 375, nota 6), venne "in minor forma riedificata alle falde del monte stesso" (G. COGNOLATO, Saggio di..., p. 52).

${ }^{25}$ A. GLORIA, Codice..., I, n. 94.

${ }^{26} \mathrm{P}$. Vedovetto in questo volume.

${ }^{27}$ A. GLORIA, Codice..., I, n. 94. Il placito costringe l'abate di Santa Maria della Vangadizza a rinunciare a metà della corte in favore del monastero veneziano di San Zaccaria.
} 

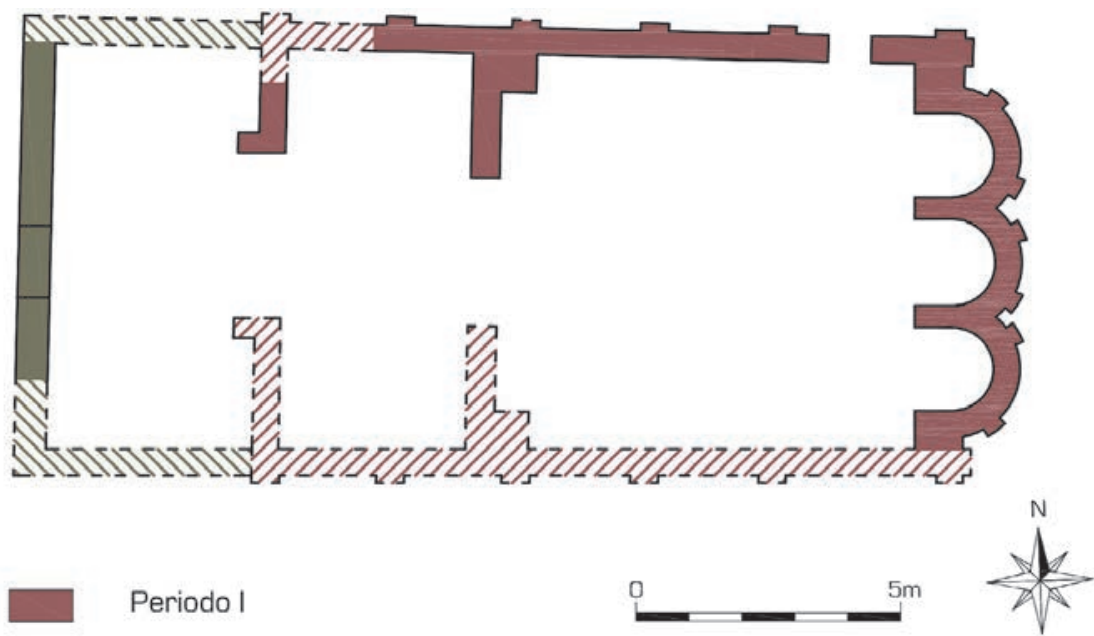

Periodo II

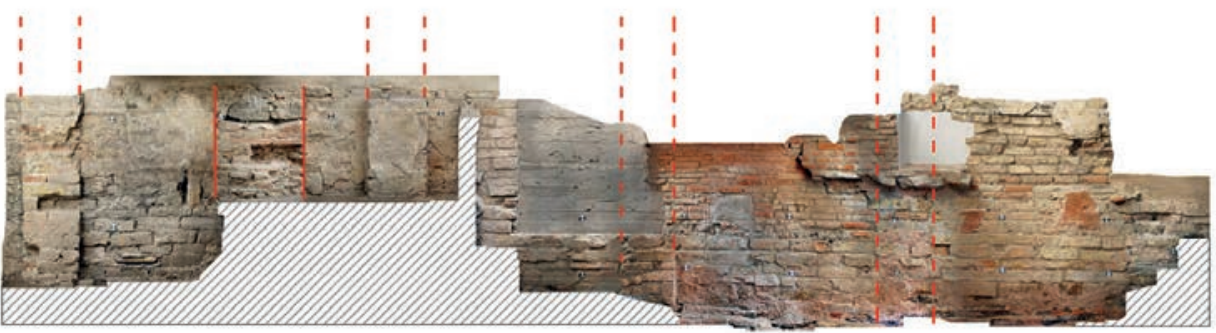

parete nord

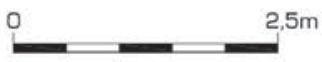

Fig. 7. Monselice, San Paolo.

A mezzacosta del versante occidentale della collina, nell'area dell'attuale villa Duodo eretta nel 1595, sorgeva il sacello eremitico di San Giorgio $\left(1099^{28}\right)$. Marin Sanudo scrive che in una arca marmorea quam ego vidi, ut dicitur, è il corpo di S. Zorzi. Nella Visita del 1489 il complesso viene descritto come un luogo angusto di 4 x 6 piedi (solo m 1,43 $\mathrm{x} 2,14$; se per errore sono stati indicati piedi anziché passi avremmo una dimensione più corretta di $\mathrm{m} 6,95$ x 10,43) e con affreschi relativi alla vita eremitica.

Sempre all'esterno della seconda cinta, in adiacenza all'area occupata ora da Ca' Marcello, dove nel XII-XIII secolo sono ricordati il palazzo pubblico, sede dei rappresentanti del potere imperiale ${ }^{29}$, la casa degli arimanni e poi quella del Comune $^{30}$, si trovava la chiesa di San Paolo, cappella dipendente dalla pieve di Santa Giustina, ma con cura d'anime per circa la metà degli abitanti di Monselice. Funzione che la mette in relazione con l'espansione altomedievale ai piedi del Colle.

Negli atti della visita pastorale il vescovo Barozzi nel 1489 descrive la chiesa a due navate, divise da tre arcate fondate su pilastri laterizi; successivi sono l'aggiunta di un'ulteriore terza navata verso nord (XVI secolo) e la ricostruzione degli inizi del XVIII.

Lo scavo degli anni '9o ha messo in luce una sequenza con quattro fasi medievali:

1. La chiesa più antica, ad aula di $\mathrm{m}$ $7,43 \times 8,63$, terminava con tre absidi (centrale: larghezza m 1,65 x 1,50 di raggio; nord: 1,60 x 1,14; sud, larga 1,58) ed era preceduta da un atrio largo $\mathrm{m}$ 3,51. Di questa chiesa si conservano le tre absidi, rinforzate da due lesene esterne, il perimetrale nord pure con lesene di $\mathrm{cm} \mathrm{50/55} \mathrm{sporgenti} \mathrm{una} \mathrm{quindicina}$ di $\mathrm{cm}$, un tratto del settore nord della facciata interna e dell'atrio. Lo spessore dei perimetrali, costruiti in laterizi in corsi sigillati da stilature a sezione trapezoidale sporgenti ca. un centimetro, è poco più di $50 \mathrm{~cm}$, ma gli angoli interni della navata sono ulteriormente rinforzati da pilastri di ca. $80 \mathrm{~cm}$. Oltre che in facciata, vi era una porta sul lato nord, in prossimità dell'abside.

La pianta, pur se utilizzata anche in seguito, è peculiare di edifici di fondazione regia soprattutto di età longobarda e carolingia ${ }^{31}$. Una datazione a fine VIII - inizi IX secolo è suggerita dalla posteriorità rispetto a strati con materiali che arrivano fino alla metà dell'VIII secolo.

2. In una seconda fase (post 8oo-ante XII secolo) la chiesa, demolito l'atrio, sarebbe stata ampliata verso ovest fino alla facciata attuale, raggiungendo una lunghezza interna complessiva di $\mathrm{m} 16,85$. Anche le murature di questa fase utilizzano laterizi romani per l'alzato, ma le fondazioni sono in blocchi di pietra di reimpiego. Di una porta centrale in facciata si intravvede lo stipite nord in laterizi, con soglia più alta di ca. $75 \mathrm{~cm}$ rispetto alla risega di fondazione della prima fase.

3. Nel XII secolo, impiegando nei paramenti masselli di pietra, oltre a laterizi provenienti dalla demolizione del perimetrale nord della prima chiesa, viene aggiunta una navata, collegata a quella esistente da arcate su pilastri. La navata termina ad est con una cappella chiusa da un muro e con una porta al centro verso l'aula. L'ispessimento delle murature nell'angolo nord est della navata e una finestrella a feritoia in facciata suggeriscono che qui vi fosse la torre campanaria. Un'iscrizione su tabella plumbea $(\mathrm{cm} \mathrm{13,} 30 \mathrm{x}$ 8,20 ) che ricorda le reliquie di Sabino (Hic ossa s(an) $c(t)$ i Savini e(pisco)pi et martiris q(ui)escunt), datata ai secoli $\mathrm{XI}-\mathrm{XII}{ }^{2}$, è probabilmente da mettere in relazione con l'ampliamento della chiesa verso ovest.

\footnotetext{
${ }^{28}$ G. COGNOLATO, Saggio di..., pp. 53, 57 .

${ }^{29}$ S. BORTOLAMI, Monselice 'oppidum opulentissimum': formazione e primi sviluppi di una comunità semiurbana del Veneto medioevale, in A. RIGON (a cura di), Monselice: storia..., pp. 101-172 e G. RIPPE, Padoue et son contado, Roma, 2003.

${ }^{30} \mathrm{~S}$. BORTOLAMI, Monselice 'oppidum opulentissimum'..., pp. 113-114.

${ }^{31}$ S. LOMARTIRE, Riflessioni sulla diffusione del tipo “Dreiapsidensaalkirche” nell'architettura lombarda, in L'édifice cultuel entre les périodes paléochrétienne et carolingienne, Zagreb, 2003, pp. 417-432.

${ }^{32}$ F. DE RUBEIS, scheda, in A. NANTE (a cura di), Santa Giustina e il primo cristianesimo a Padova, catalogo della mostra (Padova, Museo Diocesano, 27 novembre 2004-27 febbraio 2005), Casalserugo, 2004, p. 43.
} 
4. In una quarta fase (fine XIII-inizi XIV secolo, sulla base di un affresco), nell'area presbiteriale della prima chiesa viene ricavata una cripta, suddivisa in tre campate e con abside centrale che corrisponde alla preesistente.

All'esterno della cinta fortificata di VI secolo, che corre a mezzacosta, sono documentate altre tre chiese altomedievali.

San Martino in Valle, dipendente dal monastero di Santa Giustina di Padova, è stato costruito lungo la strada principale, prope pede castro ${ }^{33}$, in un'area rurale cum casis et massaritiis che nel 970, quando viene ricordata per la prima volta, era ancora all'esterno dell'abitato ${ }^{34}$. Nella Visita del 1489 ne viene ricordata la pianta irregolare, con parete sud che segue l'andamento del terreno, per cui la navata è larga m 12,15 (7 passi) verso est e 9,55 (5 passi e mezzo) verso ovest, per una lunghezza di m 19,10 (11 passi). L'abside (ad occidente?) è rettangolare di $\mathrm{m} 5$ di lato (14 piedi) con un'altezza di m 3,20 (9 piedi) fino all'attacco della volta.

Ricostruita nel XVIII secolo, delle fasi più antiche si conservano, nel lato sud, un piccolo tratto di muratura, forse altomedievale, con un paramento in pietre di cava sbozzate e laterizi di tipo romano di riutilizzo che ricorda quello di San Tommaso (infra) e il tratto inferiore del perimetrale verso monte, caratterizzato da un paramento in masselli messi in opera in corsi regolari, attribuibile ad un rifacimento romanico (XII secolo). L'asimmetria notata dal Barozzi potrebbe dunque essere il risultato di queste due fasi costruttive.

Santo Stefano, orientato a nord, era priorato di Nonantola (poi viene attribuita ai frati conventuali). Nella visita del 1489 viene descritta a due navate, divise da tre arcate sostenute da quattro pilastri, comprese in questi due semicolonne: mentre la navata di destra è di m 5,21 (3 passi) e di simile altezza, l'altra di m 10,43 (6 passi) si allarga poi in un transetto (di 4 X 10 passi) che sporge, rispetto a questa navata, di m 3,47 (2 passi) e si prolunga per m 6,95 (4 passi). L'abside è un quadrato di m 5,71 di lato concluso ad est da un pentagono e presenta inoltre un arco a fasce (in morem fassie).

Da questa descrizione sembra dedursi una prima chiesa con pianta a T, alla quale si aggiunge in seguito una seconda navata verso sud.

San Tommaso, sempre ai piedi del castello (retro muris de ipso castello), ma sul lato nord est, era chiesa con cura d'anime della corte di Petriolo, ubicata su un dosso circolare in prossimità del vecchio percorso del fiume Vigenzone. Nel 911 il conte di Verona Inghelfredo dona al monastero veneziano di San Zaccaria le corti di Petriolo e Cona, da lui ottenute otto anni prima (nel 906) dal vescovo veronese Adelardo35. La chiesa è stata ristrutturata nel XVII secolo, quando l'aula absidata è stata affiancata da due cappelle laterali a pianta rettangolare e dalla sacristia. Nella visita pastorale del vescovo Barozzi la chiesa, ubicata in calcinara sub castro sancti Georgi, viene descritta a due absidi con una finestra ciascuna, entrambe provviste di un altare, mentre un terzo altare è stato addossato alla muratura tra le due absidi. Alla chiesa ricordata nel X secolo, ma probabilmente più antica, è riferibile la muratura, visibile negli angolari est, realizzata in trachite e mattoni romani di riutilizzo, alcuni dei quali, di grosso modulo, sono simili a quelli reimpiegati nella chiesa di San Paolo. A questa fase costruttiva appartiene anche un'abside semicircolare conservata per uno-due corsi, messa in luce durante i restauri degli anni ' 80.

\section{(d) chiese nelle campagne di Monselice}

All'esterno della cinta di XIII secolo, e dunque in una fascia che in età altomedievale era rurale, sono attestati dalla fonti scritte altri cinque luoghi di culto.

Il monastero di San Salvatore (1164), ad un tiro di freccia dalla porta orientale di Monselice, nel 1489 (Visita Pastorale del Barozzi) dipendeva da Santa Giustina di Padova, che lo usava (in celle abitate da 3-4 sacerdoti), come residenza estiva o per convalescenza da malattia o propter nimis diuturna clausura dei monaci che avevano necessità di mutare aerem vel laxare animum. La chiesa misurava m 13,90 x 5,71 (= passi 8 per 16 piedi), era alta $m$ 4,30 (12 piedi) fino all'inizio della volta che, suddivisa in tre ordini, la copriva interamente. La calotta dell'abside, ad oriente, aveva la forma di mezza sfera. Addossata alla facciata vi era una loggia sopraelevata, che occupava quasi tutta la terza volta, dalla quale i monaci potevano assistere alla messa. Vi erano due porte, una a nord e l'altra a sud che metteva in comunicazione con il monastero, e altrettante finestre nei medesimi lati. Accanto alla chiesa sorgevano numerosi edifici.

La chiesa di San Daniele, priorato dipendente dall'abbazia di Nonantola, nella Visita Barozzi del 1489 viene descritta ad aula unica m 10,43 x 17,40 (passi 6 x 8) con abside rettangolare di $\mathrm{m}$ 5,21 di lato ed altare addossato alla parete abside. Altre informazioni aggiunge il Furlani in un manoscritto del 1848: (a) "quasi tutta demolita nell'anno 1835 era tutta d'architettura gotica, specialmente il campanile ancora esistente"; (b) "alle pareti d'un pezzo di muraglia lasciata in piedi un frammento d'antica lapide con lettere all'uso longobardico"; (c) scelta come cimitero dei morti di peste, il che fa ipotizzare fosse all'esterno dell'abitato; (d) due epigrafi, datate 1424, del rector Benedictus Moraelius, la prima funeraria, la seconda nella cappella di Santa Maria hoc opus fecit fieri; (d) il San Daniele del titolo sarebbe il santo le cui reliquie sono state scoperte nel 1075 nella chiesa di Santa Giustina, per cui la chiesa di età longobarda avrebbe avuto un diverso titolo ${ }^{36}$. Servono ulteriore ricerche per appurare se questa informazione è corretta e non si tratti invece del San Daniele profeta, oggetto di dediche anche in età longobarda.

Altre chiese suburbane erano: San Lorenzo lontana da Monselice un tiro di freccia (Visita Barozzi 1489: $\mathrm{m} \mathrm{12,15} \mathrm{X}$

\footnotetext{
33 a. 1014: A. GLORIA, Codice..., II, n. 98.

34 A. GLORIA, Codice...,I, n. 55.

${ }^{35}$ A. GLORIA, Codice..., nn. 26, 29, p. 46. K. MODZELEWSKI, Le vicende della "pars dominica"nei beni fondiari del monastero di San Zaccaria di Venezia (sec. X-XIV), in Bollettino dell'Istituto di storia della società e dello stato veneziano, IV, 1962, pp. 42-79; G. TASINI, Monselice e il monastero di S. Zaccaria di Venezia, in A. RIGON (a cura di), Monselice nei secoli..., pp. 189-199; M. BOLZONELLA, L'espansione patrimoniale di enti religiosi e monastici veneziani nel territorio padovano (sec. X in.-XIV ex.), Tesi di dottorato, 2011, p. 24.
}

${ }^{36}$ A. F. Furlani, Storiche Notizie di Monselice, 1848, Biblioteca Comunale di Monselice, ms. 10017, 10018, 10019, 
$17,40=7$ x 10 passi); San Sebastiano, lungo la strada per Este, nella visita del 1489 è definita come hospitale, occupato da un certo Francesco Verzeleso qui in eo non tenet aliquam hospitalitatem; San Vito, ai piedi del Monte Ricco, di m 6,95 per lato, alta fino al tetto 12 piedi, con ad oriente un'abside semicircolare; già sede di un eremita, al tempo del Barozzi era di proprietà dei Carmelitani installati nella vicina chiesa di Santa Maria del Pilastro; San Giovanni Battista, sulla sommità del Monte Ricco, un tempo oggetto di grande venerazione, nel 1489 risultava diroccata. Costruita interamente in pietra misurava m 10,43 x 6,95 (6x4 passi) per un'altezza di 3 ed aveva una piccola abside quadrata di m 3,48 per lato ${ }^{37}$ nella quale si trovava l'altare, alla distanza di 4 piedi dalla parete.

Infine, dipendenti dalla Pieve di Monselice, erano, nel 1489, Santa Maria di Pozzonovo, San Bartolomeo nella contrada omonima, Santa Maria e Santa Maria Nove Sclavonie a Solesino, Sant'Elena nella località omonima, San Nicolò di Marendole, San Matteo di Vanzo. Degna di nota è anzitutto la chiesa dei Santi Cosma e Damiano, costruita su un paleodosso a 2,6 km (1500 passi) da Monselice e ricordata alla metà del Duecento ${ }^{38}$. Ad aula unica, di m 6,95 x 13,90 (4 X 8 passi), con abside orientata, aveva una recinzione presbiteriale (definita semplicemente come "parete"). Addossati alla recinzione vi erano due altari. A sud della chiesa si tro- vavano la sacrestia e una domus. San Michele di Baguarolo, prope Montemsilicis (ricordata nel 1191), il Barozzi la ricorda diroccata con tre absidi e tre altari (dunque ad aula unica).

In conclusione, nella costruzione della rete ecclesiastica di Monselice possiamo ipotizzare: (a) una prima fase con la fondazione di chiese in relazione all'insediamento di età romana; (b) una seconda con la pieve di Santa Giustina, contestuale alla costruzione del castello nel VI secolo; (c) un terza, altomedievale, con il moltiplicarsi delle fondazioni sia all'interno del castello, sia all'esterno, comunque entro il perimetro del monte, sia nella campagne circostanti e sul Monte Ricco.

\section{MONTAGNANA E LA PIEVE DI MEgLIADINO SAN FIDENZIO}

La prima attestazione di Montagnana è nella donazione del marchese Ugo di Toscana, nel 996, quando viene indicata come curte domnicata cum castello et ecclesia inibi consistente. Il castello era peraltro condiviso con gli uomini di Altadura, Casale, Urbana e San Salvaro, comunità della Scodosia che vi prestavano la fidelitas (riconoscimento della dipendenza giurisdizionale), contribuivano alla custodia e in cambio vi potevano "incanevare" e "incastellare" sicut castrum de Scodescia ${ }^{39}$. Questi obblighi derivavano probabilmente da un'originaria condizione di arimanni,

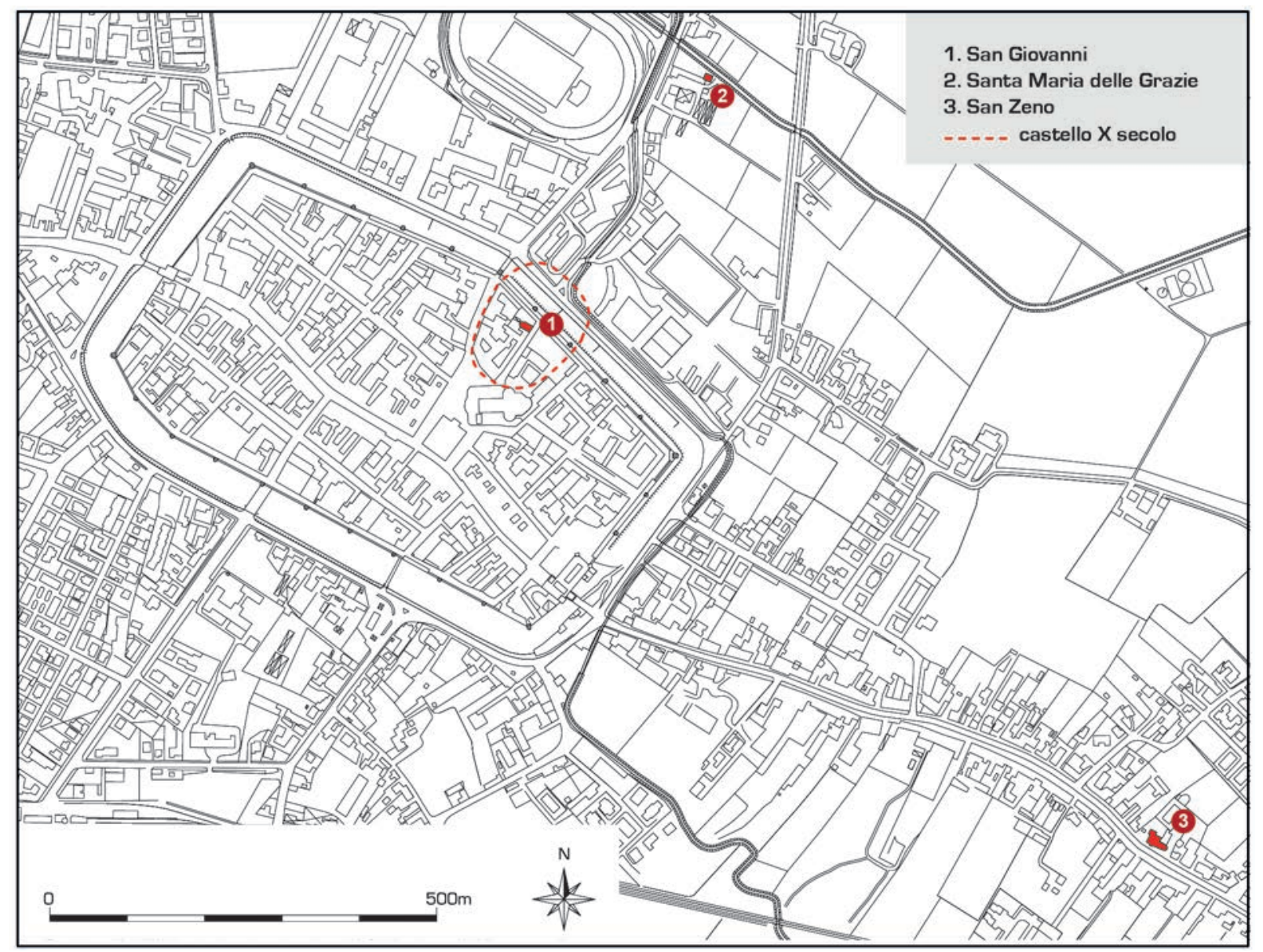

Fig. 8. Montagnana, posizione dell'abitato, del castello e delle probabili chiese altomedievali.

\footnotetext{
37 Nella trascrizione 2 x 2 piedi $=\mathrm{m}$ o,71 di lato, ma credo sia un errore per passi così come per l'altezza fino all'attacco della volta.

${ }^{3}$ S. BORTOLAMI, Monselice 'oppidum opulentissimum'..., p. 122, nota 129.

${ }^{39}$ E. ZORZI, Il territorio padovano nel periodo di trapasso da comitato a comune. Studio storico con documenti inediti, in Miscellanea di storia veneta della Deputazione di storia patria per le Venezie, s. IV, 5, Venezia, 1931, pp. 181-194 e doc, IV, pp. 271-286; S. BORTOLAMI 2006, Montagnana nel Medioevo: nascita di una città murata, in L. OLIVATO, E.M. DAL POZZOLO (a cura di), Montagnana. Storia e incanto, Cornuda (Tv), pp. 41-42.
} 


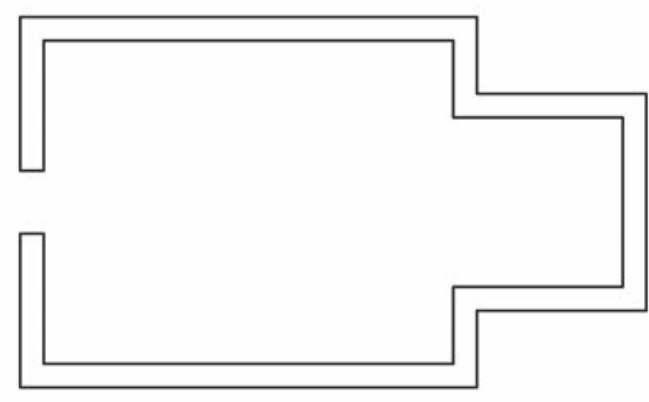

San Giovanni Battista

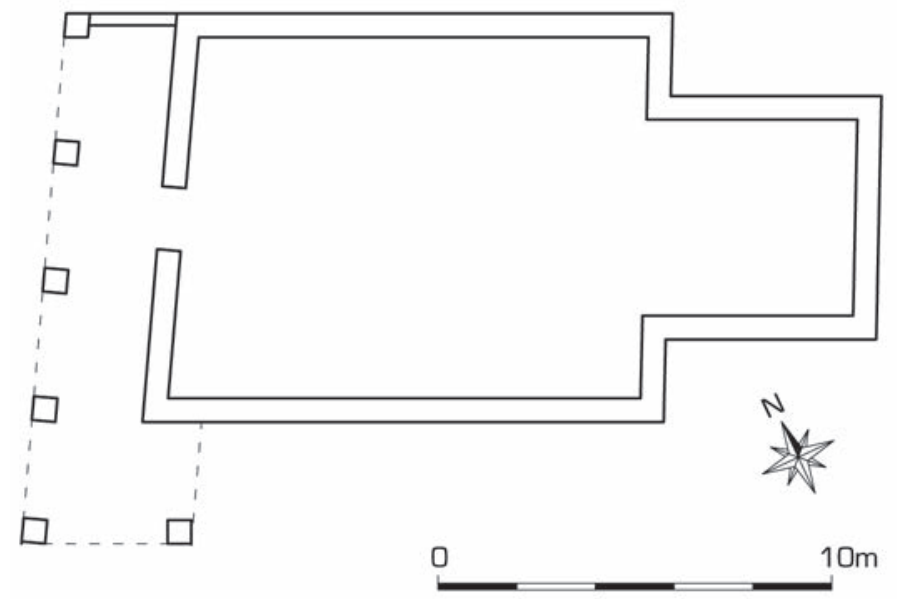

San Zeno

Fig. 9. Montagnana, San Giovanni Battista e San Zeno, pianta ricavata dalla descrizione del vescovo Barozzi (1489).

nell'ambito del distretto della Scodosia, forse costituito in età longobarda ${ }^{40}$. In assenza di ricerche, testimonianze di quel periodo sono per ora costituite dal solo toponimo Stoegarda (recinto per cavalli) ${ }^{41}$ e da tre chiese di probabile origine altomedievale (figg. 8, 9).

La chiesa di Santa Maria, ricostruita a cavallo dell'abitato più antico nella seconda metà del XV secolo ${ }^{42}$, dipendeva dalla pieve di San Fidenzio di Megliadino, il che fa pensare che Montagnana non fosse un centro importante prima della fine dell'XI secolo, quando vi si installano i marchesi d'Este.
All'interno della cinta duecentesca, si riconosce però un nucleo semicircolare, la cui parte nord è stata tagliata dalle mura. In quest'area si trovava la chiesa di San Giovanni, ricordata nel testamento di Azzo d'Este del 1142. Nella visita pastorale del Barozzi si specifica che misura $\mathrm{m} \mathrm{8,20} \mathrm{x} \mathrm{10,40}$ (23 piedi per 6 passi), con un'abside quadrata di $\mathrm{m} \mathrm{4,30} \mathrm{di}$ lato (12 piedi).

La chiesa di San Zeno, giuspatronato del Comune, ha dato il nome ad un sobborgo, ad un ponte e ad una palude; attestata nel 1073 è raffigurata in una mappa del 1792. Ad aula unica con abside rettangolare, nell'angolo sud ovest aveva un campanile romanico alto ben $20 \mathrm{~m}$, demolito nel $1905^{43}$.

Entrambe le intitolazioni e l'abside rettangolare si ritrovano in molte chiese altomedievali, ma nel nostro caso solo uno scavo potrebbe confermarlo. Da una di queste due chiese, o dalla parrocchiale di Santa Maria, proviene probabilmente un frammento di pluteo altomedievale riutilizzato come acquasantiera nella chiesa suburbana di Santa Maria delle Grazie (Vedovetto in questo volume).

La parrocchia di Montagnana dipendeva, come abbiamo visto, dalla pieve di San Fidenzio di Megliadino che era dunque, almeno dal punto di vista ecclesiastico, più importante e lo suggerisce anche lo splendido ambone della metà dell'VIII secolo, pertinente alla chiesa di San Tommaso, titolo originario di quella chiesa, la cui storia è assai interessante pur se ricostruita su base indiziaria con le sole fonti scritte ed architettoniche (fig. 10).

Un documento del 7 giugno del $978^{44}$ attribuisce al vescovo padovano Gauslino la rifondazione, come collegiata, della chiesa di San Tommaso, nella quale aveva fatto trasferire il corpo di s. Fidenzio, rinvenuto durante lavori intrapresi nel bosco di Polverara, un centro della Saccisica distante più di trenta chilometri ${ }^{45}$.

Che la chiesa di San Tommaso risalga all'età longobarda lo suggeriscono il lettorino di ambone e l'iscrizione di un sarcofago (VIII secolo: Vedovetto in questo volume), la dedica che si ritrova nella chiesa della curtis fiscale di Corte di Piove di Sacco e in quella di Petriolo e la presenza di arimanni, ricordati nella donazione di Enrico IV del 1077 per Folco e Ugo, figli del marchese Alberto Azzo II, fondatore della casa estense.

La chiesa di San Fidenzio, descritta nel 1489 dal vescovo Barozzi, misurava ca. $\mathrm{m} 27,40 \times 17,50$, era a tre navate (larghe, la centrale, $\mathrm{m} 6,95$, le laterali $\mathrm{m} 5,20$ ) con un'abside semicircolare centrale collegata ad un coro sopraelevato al quale si saliva mediante una scala di ben 10 gradini (ca. due metri), larghi quanto la navata centrale. Due colonne ai lati della

\footnotetext{
${ }_{40} \mathrm{Nel} 1077$ l'imperatore Enrico IV conferma, infatti, a Folco e Ugo le arimannie di Casale, Altaura ed Urbana (MGH, DD Heinrici IV, n. 289).

${ }^{41}$ Archivio di Stato di Padova, Giustinian 5174, a. 1318; sul significato G.B. PELLEGRINI 1987, Ricerche di toponomastica veneta, Padova, p. 332.

${ }^{42}$ I. DANIELE, La diocesi di Padova, Padova, 1973, p. 363; era in costruzione nel 1489, al tempo della visita pastorale del Barozzi.

43 A. BORIN, Note di storia montagnanese, Montagnana, 1990, p. 145.

44 B. LANFRANCHI STRINA, SS. Trinità e S. Michele Arcangelo di Brondolo. II. Documenti 8oo-1199, in Fonti per la Storia di Venezia, Sez. II, Archivi ecclesiastici, Diocesi Clodiense, Venezia, 1973, pp. 139-141. Il documento conferma ed integra l'iscrizione del 1685, apposta in occasione di un restauro (TEMPLUM HOC SACRUM EX CAMPESTRI/ SACELLO D. OLIM APOSTOLO THOMAE DICATUM/ IN HANC AMPLIOREM FORMAM AB ILUSTRISSIMO ET REVERENDISSIMO GAUSLINO TRANSLAGARDO/ EPISCOPO PATAVINO CONSTRUCUTM DIVOQUE FIDENTIO/ EX VOTO DICATUM, IN COLLEGIALEM/ ERECTUM ET CIRCA ANNUM NONGENTESIMU/ CONSECRATUM FUIT... (I. DANIELE, La diocesi..., 1973, p. 343). Reliquie di San Fidenzio servirono anche per la consacrazione delle chiese presso il castello di Baone e di Sarmeola (attestata nel 113o, tra le pertinenze dei canonici della cattedrale: A. GLORIA, Codice..., II, n. 212; I. DANIELE, La diocesi..., p. 622). Altre chiese con reliquie di San Fidenzio si trovano a Pernumia, in terratorio ubi dicitur Cornedo (a. 1181: A. GLORIA, Codice..., II, n. 1403), Roncaiette e Fornace (a. 1297, P. SELLA, G. VALLE (a cura di), Rationes decimarum Italiae nei secoli XIII-XIV. Venetiae-Histria Dalmatia, Città del Vaticano, 1941, n. 1722).

${ }^{45}$ A. GLORIA, Il territorio...,III, p. 279; Gennari la pone nel 1005 (G. GENNARI, Annali della città di Padova, II, Bassano, 1804, p. 8); sul luogo del rinvenimento venne edificata una prima chiesa in suo onore, attestata nel 1130: G. BRUNACCI, Storia Ecclesiastica di Padova, 1755, p. 863. G.P. MASIERI, Memorie antiche ecclesiastiche e profane sulla città e diocesi di Padova, II, Padova, 1801, p. 254, citato da A. GLORIA, Il territorio...,III, p. 279.
} 

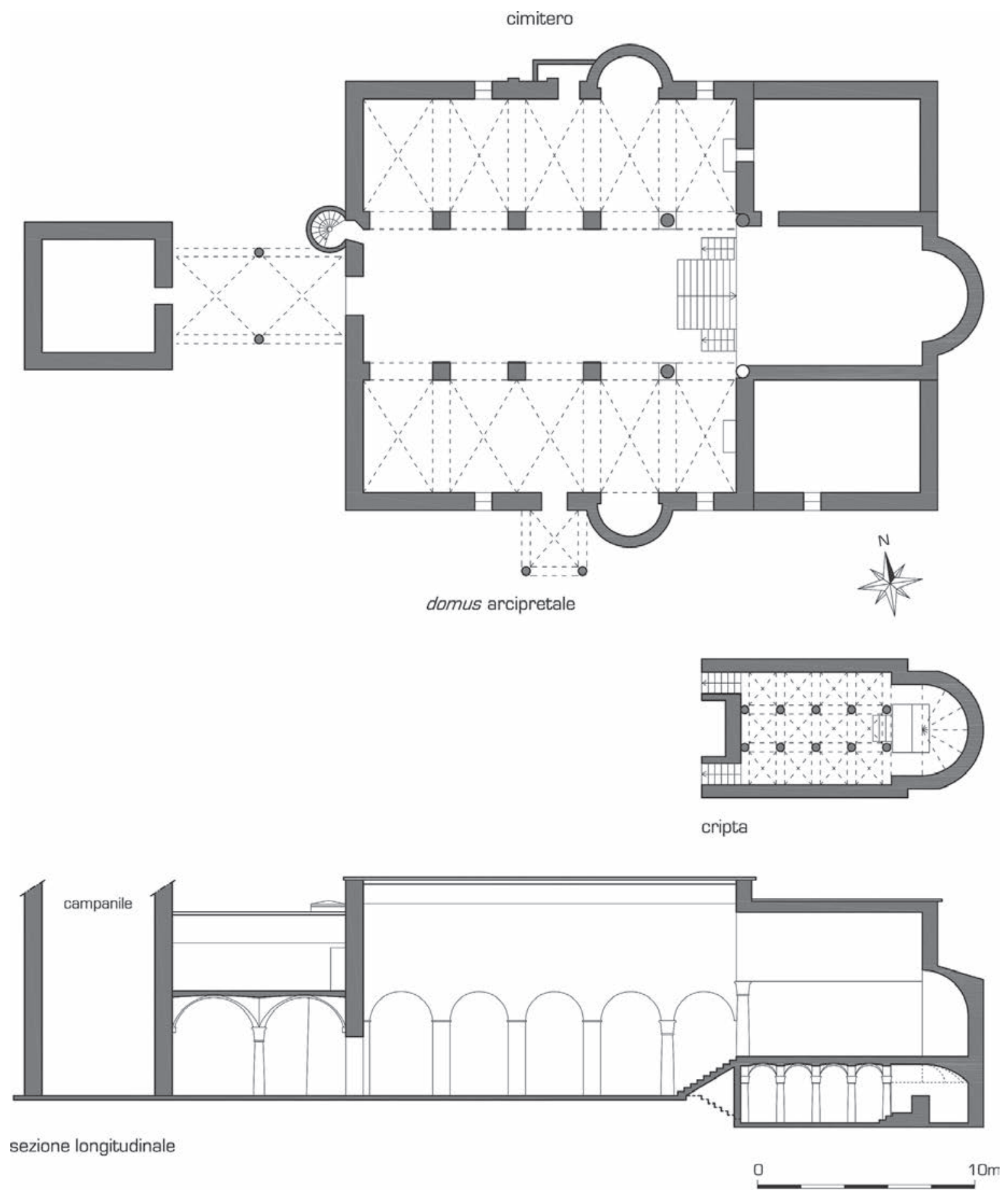

Fig. 10. Megliadino, San Fidenzio, pianta ricavata dalla descrizione del vescovo Barozzi (1489).

scala (alla base alte m 3,47 e in alto di metà altezza) e tre pilastri per parte (con un intervallo di $\mathrm{m} 3,47$ ) sostenevano i divisori tra navata centrale e laterale.

Sotto il coro vi era la cripta, alla quale si scendeva con due scale in marmo di 6 gradini ciascuna. Larga m 5,21 e lunga m 6,95, aveva un'abside semicircolare (evidentemente sottostante quella del coro) ed era ripartita, in tre navatelle di pari ampiezza, da cinque colonne per lato, due delle quali erano di marmo pregevole; prive di basi erano alte $\mathrm{m} \mathrm{2,14}$, compresi i capitelli, e sorreggevano le volte. Il corpo di San Fidenzio era inumato in un'arca di pietra satis elegans; posizionata nella navatella centrale della cripta (ma ad orientem respicente) era sostenuta, a parte posteriore, da due colonne alte $90 \mathrm{~cm}$, mentre sul davanti poggiava probabilmente sulla muratura di una scala di tre gradini di pietra. Le volte della cripta erano affrescate.
Davanti alla chiesa, secondo il Barozzi, a 5,20 di distanza, vi era un possente campanile, collegato alla chiesa da un edificio con portico a due campate voltate con colonne al centro e soprastante loggia, accessibile, dall'interno della chiesa, tramite una torre scalare circolare addossata alla facciata. A nord si trovava il cimitero, a sud il cortile della domus arcipretale.

In mancanza di dati materiali, risulta arduo proporre una sequenza e una datazione del complesso dettagliatamente descritto dal Barozzi. La pianta inconsueta, con il coro che sporge di oltre $8 \mathrm{~m}$ rispetto alle navate, potrebbe essere il risultato del recupero della chiesa di San Tommaso come cripta, alla quale è stata aggiunta l'aula a tre navate. Per quanto riguarda la datazione il confronto della nostra cripta con quella di età ottoniana di San Vincenzo di Galliano suggerisce sia opera di Gauslino. 

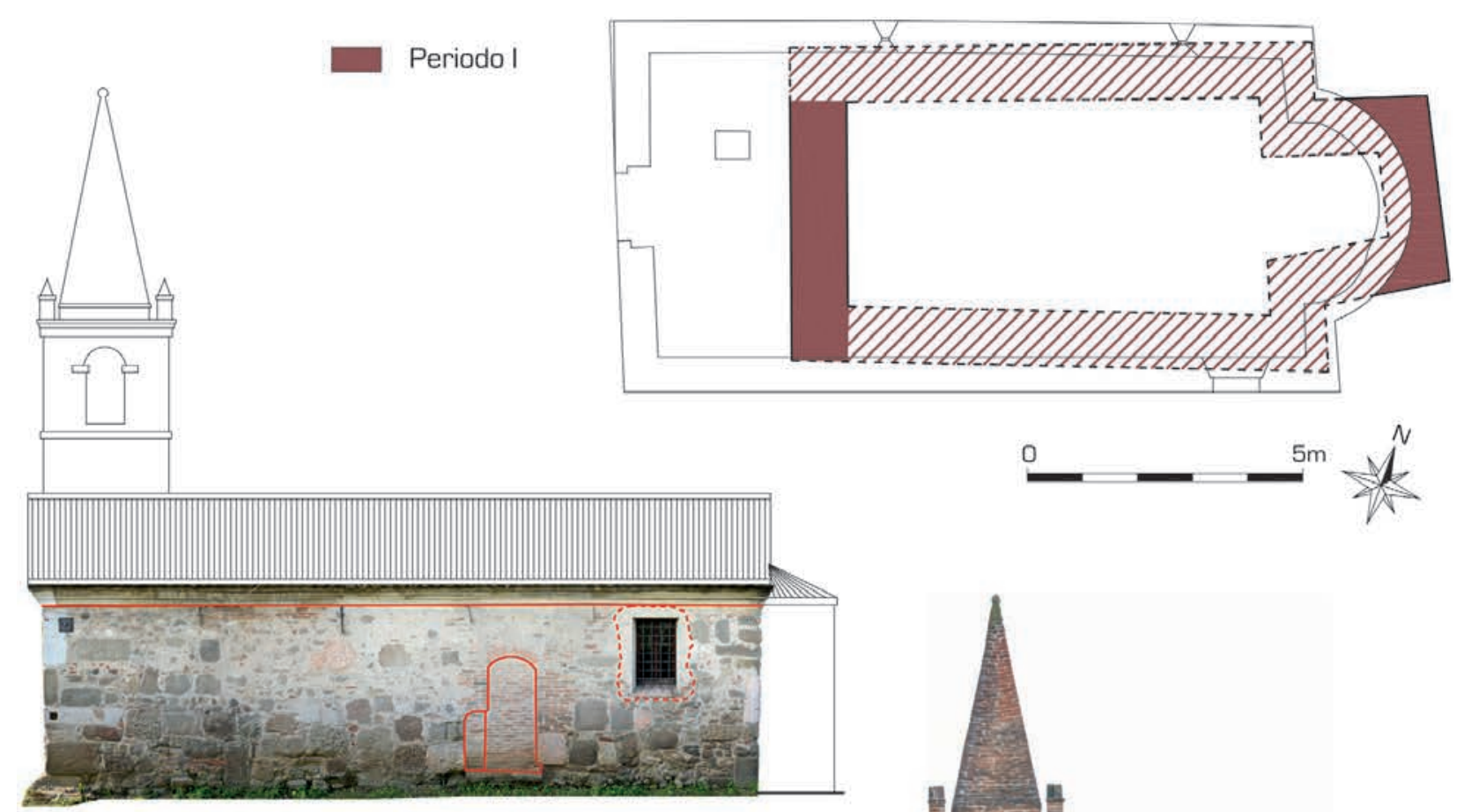

prospetto sud

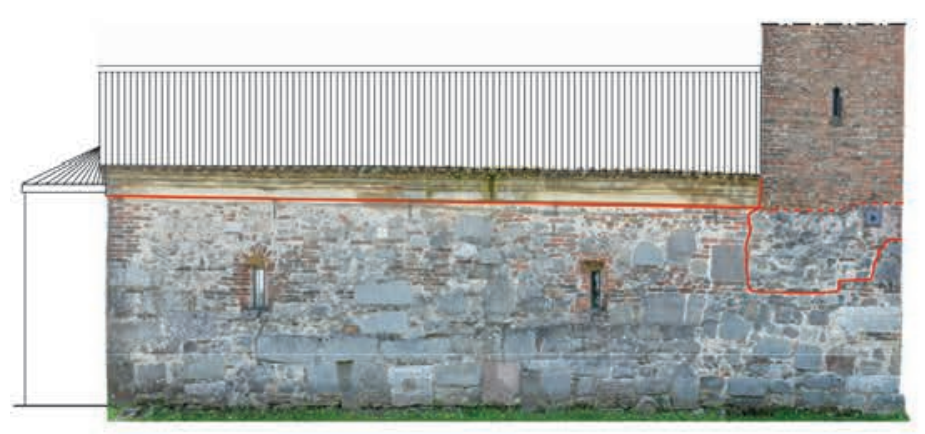

prospetto nord

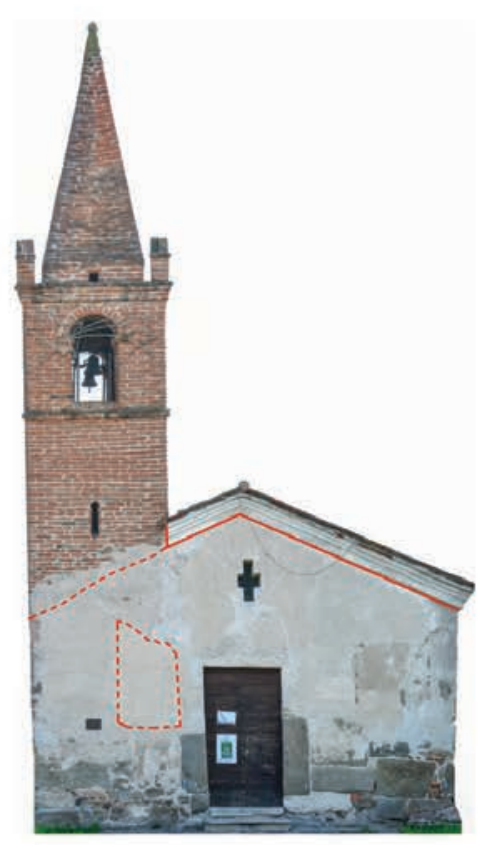

prospetto ovest, facciata

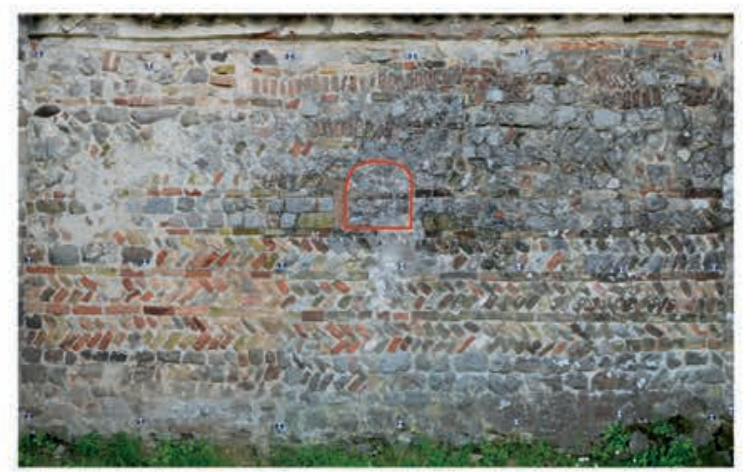

abside

o

$2.5 \mathrm{~m}$

Fig. 11. Saletto, San Silvestro.

Dalla pieve di San Fidenzio dipendeva anche San Silvestro di Saletto. Nella visita pastorale del Barozzi del $1488 \mathrm{si}$ specifica essere stata una ecclesia hospitalis, la prima ecclesia dicti loci ${ }^{46}$. Ricordata per la prima volta il 27 febbraio del $1145^{47}$, si conserva ora nella ricostruzione romanica ad aula unica di m 6,6o x 12,70 con abside semicircolare orientata ad est (fig. 11). Nella parte inferiore dei perimetrali laterali e della facciata sono state reimpiegate grosse pietre squadrate con alcuni elementi architettonici e laterizi frammentari di età romana. Nella parte alta dei perimetrali laterali, oltre ad alcune pietre squadrate di recupero, vi sono invece laterizi bassomedievali di piccolo modulo, con i quali sono state

${ }^{46}$ I. DANIELE, La diocesi..., p. 496.

${ }^{47}$ I. DANIELE, La diocesi..., pp. 495-496. 
realizzate anche le cornici delle finestre strombate (una rettangolare, l'altra ad arco). Nell'abside, dal basso verso l'alto, si susseguono alcuni corsi in pietre spaccate e alcune bozze, quattro fasce di opera listata con filari di laterizi di recupero romani, disposti in orizzontale e a spinapesce. Nelle condizioni attuali di lettura, si notano: (a) un'evidente posteriorità sia della finestra aperta nel lato sud in corrispondenza dell'abside, sia degli intonaci in facciata che rivestono più fasi di affresco conservati in frammenti; (c) un rifacimento della parte alta dell'abside, con tamponamento di una finestrella ad arco al centro, intuibile da un laterizio messo in verticale e dall'accenno dell'arco soprastante; (d) una probabile posteriorità della porta ad arco ribassato e bardellone, con cornice in laterizi bassomedievali, aperta nella parete sud. A sua volta, l'eterogeneità delle murature potrebbe derivare da una ricostruzione generale, nel XII secolo, che ha interessato la parte alta della chiesa e il campanile, dove sono impiegati esclusivamente laterizi medievali, ovvero essere il risultato dell'impiego nelle parti più basse dei materiali provenienti dall'edificio precedente.

Gli scavi conclusi nel 1999 hanno messo in luce una chiesa più antica (VII secolo?), larga uguale, ma più corta e leggermente più piccola e con abside rettangolare $4^{48}$, La pianta, le modeste dimensioni, le poche sepolture (tre all'interno, sei davanti alla facciata) la qualificano come chiesa funeraria privata.

\section{CONCLUSIONI}

Il territorio del basso corso dell'Adige è tra quelli più soggetti alle trasformazioni ambientali, a causa del dissesto idrogeologico del VI secolo, al quale potrebbe aver concorso anche la frammentazione del controllo del fiume tra Longobardi e Bizantini. E tuttavia gli effetti furono variegati: a Montagnana e nell'area centuriata le campagne romane sono state sepolte da alcuni metri di detriti alluvionali, mentre attorno a Monselice le quote sono rimaste pressoché invariate. La sostituzione, come capoluogo distrettuale, di Este con Monselice, sembra derivare da motivazioni strategiche: più vicina a Padova poteva meglio fronteggiare la costa rimasta bizantina.

In questo contesto non sorprende la distribuzione delle chiese e dell'arredo liturgico nei centri collocati lungo l'antico percorso dell'Adige: oltre ad una continuità demica, vi si concentravano infatti le sedi di potere. Assai significative sono, da un lato, la trasformazione policentrica di Este che aveva perso la status di città, dall'altro la costruzione, a Monselice, di una topografia cristiana degna di una città, quale era in effetti diventata in quanto sede di gastaldaga. In entrambi questi centri, come in altri collegati al potere, è dunque naturale trovare testimonianza di chiese e di arredo liturgico altomedievale.

Resilienza, adattamento, sviluppo ed evergetismo religioso si collocano nei punti nevralgici, veri e propri porti, di un percorso fluviale che collegava l'Adriatico con un'importante città qual era Verona: i commerci di prodotti essenziali, come il sale e le spezie, e di merci di lusso non sono venuti mai meno, seppure con numeri che solo dalla fine del X secolo diverranno consistenti, assicurando a Venezia i ricchi mercati della Marca di Verona, premessa di un'egemonia che si imporrà nel corso dell'avanzato XI-inizi XII secolo.

${ }_{4^{8}}$ A. COLECCHIA, S. TUZZATO, Saletto, San Silvestro (scheda), in G.P. BROGIOLO, M. IBSEN (a cura di), Corpus Architecturae... pp. 212-217. In fase di scavo è stata ipotizzata anche un'ulteriore fase, più antica, con abside semicircolare che, a mio avviso, costituisce solo la fondazione interna dell'abside rettangolare. 